University of Nebraska - Lincoln

DigitalCommons@University of Nebraska - Lincoln

Robert Katz Publications

Research Papers in Physics and Astronomy

January 1994

\title{
Survey of Cellular Radiosensitivity Parameters
}

Robert Katz

University of Nebraska-Lincoln, rkatz2@unl.edu

Rashidah Zachariah

University of Nebraska-Lincoln

Francis A. Cucinotta

NASA Langley Research Center, Hampton, Virginia, francis.cucinotta@unlv.edu

Chunxiang Zhang

Zhongshan University, Guangzhou, People's Republic of China

Follow this and additional works at: https://digitalcommons.unl.edu/physicskatz

Part of the Physics Commons

Katz, Robert; Zachariah, Rashidah; Cucinotta, Francis A.; and Zhang, Chunxiang, "Survey of Cellular Radiosensitivity Parameters" (1994). Robert Katz Publications. 39.

https://digitalcommons.unl.edu/physicskatz/39

This Article is brought to you for free and open access by the Research Papers in Physics and Astronomy at DigitalCommons@University of Nebraska - Lincoln. It has been accepted for inclusion in Robert Katz Publications by an authorized administrator of DigitalCommons@University of Nebraska - Lincoln. 


\title{
Survey of Cellular Radiosensitivity Parameters
}

\author{
Robert Katz, ${ }^{*}$ Rashidah Zachariah, ${ }^{*}$ Francis A. Cucinotta ${ }^{\dagger}$ and Chunxiang Zhang ${ }^{\ddagger}$ \\ *University of Nebraska, Lincoln, Nebraska 68588-0111; ${ }^{\dagger}$ NASA Langley Research Center, Hampton, Virginia 23665-5225; \\ and ${ }^{\ddagger}$ Zhongshan University, Guangzhou, People’s Republic of China
}

Katz, R., Zachariah, R., Cucinotta, F. A. and Zhang, C. Survey of Cellular Radiosensitivity Parameters. Radiat. Res. 140, 356-365 (1994).

A model of the formation of particle tracks in emulsion has been extended through the use of biological target theory to formulate a theory of the response of biological cells and molecules of biological importance to irradiation with energetic heavy ions. For this purpose the response to $\gamma$ rays is represented by the single-hit, multitarget model with parameters $m$ and $D_{0}$, while additional parameters $\kappa$ (or $a_{0}$ ) and $\sigma_{0}$ are required to represent the size of internal cellular targets and the effective cross-sectional area of the cell nucleus, respectively, for heavy-ion bombardments. For one-or-more-hit detectors, only the first three of these parameters are required and $m=1$. For cells $m$ is typically 2 or more. The model is developed from the concept that response to secondary electrons follows the same functional form for $\gamma$ rays and for the $\delta$ rays surrounding an ion's path. Originally applied to dry enzymes and viruses in 1967, the model of the one-hit detector has been extended to emulsions, to other physical and chemical detectors, to single- and double-strand breaks in DNA in EO buffer and to three E. coli strains. The two-hit response has been observed for "track core" effects in radiation chemistry, for supralinearity in thermoluminescent dosimeters and for desensitized nuclear emulsions, where hit numbers up to 6 have been observed. In its extension to biological cells, additional concepts are required relating to the character of the track, namely the grain-count and track-width regimes, and to the ability of multitarget systems to acquire damage from intertrack $\delta$ rays (called gamma kill) as well as from intratrack $\delta$ rays (called ion kill). The model has been applied to some $\mathbf{4 0}$ sets of radiobiological data obtained from $\gamma$, track-segment heavy-ion and neutron irradiations. Here we elaborate on the meaning of these concepts, tabulate the cellular parameters, and display their systematic behavior and the relationships among them. In particular the parameter $\kappa$, which serves to determine the location in $Z^{* 2} / \beta^{2}$ of the maximum value of the $R B E$, shows little variation among cell types, while $D_{0}$, which describes the response to $\gamma$ rays and $\sigma_{0}$, which appears to indicate the target size, varies over many orders of magnitude.

\section{INTRODUCTION}

Track physics, the study of the tracks of ionizing particles in biological (and other) matter, has sorted out several problems which troubled earlier investigators: how to treat the effects of $\delta$ rays, what is the basis of the variation of the shapes of survival curves with LET (linear energy transfer or stopping power), and what are the relative roles of energy deposition and particle fluence. It has shown that the answers to these questions are related to the physics and the structure of particle tracks as well as to biology. In this paper we remind the reader of the formulas and concepts of our model, tabulate its parameters and examine their distribution for different biological cells and end points. The entire biological content of the model resides in the choices of radiosensitivity parameters and their numerical values. That is, biological response is parameterized and not (yet) associated with causality. Dose, an average amorphous statistical macroscopic concept, is seen to be a useful plotting parameter for low-LET radiations, but of little fundamental merit otherwise. The transition from macro- to microdosimetry or from $\gamma$ rays to ultrasoft $\mathrm{X}$ rays must be considered carefully, for there are subtle alterations of meaning in these transitions similar to those involved in the transition of the concept of temperature as the dimensions of an object decrease from macroscopic to microscopic, to individual molecules, where temperature is meaningless.

To analyze the response of detectors (including biological cells) to energetic heavy ions, the model requires that we first know (or infer) their response to $\gamma$ rays as a function of dose. Next we require knowledge of the radial distribution of the dose from $\delta$ rays about the path of an average energetic heavy ion. These are combined to yield the probability for interaction with the target(s) of a detector as a function of the radial distance from the ion's path. Radial integration of this probability yields a quantity called the cross section, the probability that a beam containing a sin- 
gle ion per unit area will interact with and cause a response in a single cell contained in a unit area through which the beam passes. The cross section typically does not represent the target size, and may be identified with size only if the interaction probability is much larger when the particle passes through the target than when the projectile passes outside the target. This consideration is rarely fulfilled with one-hit detectors because of the influence of long-range $\delta$ rays, for the passage of a single electron through a one-hit target may suffice to inactivate it. As used here, "hit" refers to that which causes detector response. As a result the interaction cross section cannot be identified with target size for one-hit detectors. A response produced by the passage of a single ion through or adjacent to a target is called "ion kill." This term fully describes low-fluence irradiations where it is unlikely that $\delta$ rays from different ions in a beam will intersect in a single cell. In this case all interactions are "intratrack" in character.

At high fluence, when $\delta$ rays from different ions overlap, we must consider intertrack effects, here called "gamma kill," because it is described by the same equation used for cell killing or survival after $\gamma$ irradiation. Because of the statistical fluctuation in the production of $\delta$ rays we consider that only a fraction, $P$, of the cells intersected by an ion is killed (or mutated) in the "grain-count regime," where the track has the appearance of a random string of beads. (In nuclear emulsions such tracks are analyzed by counting the number of developed grains per unit path length.) There are gaps between inactivated cells in which cells may be damaged sublethally because of fluctuations in the production of $\delta$ rays. Cells which are not killed in the ion-kill mode, by the passage of a single ion, may be damaged further and killed by $\delta$ rays from an adjacent ion at high fluence. We make the approximation that the fraction, $P$, of the cells that are inactivated in ion kill also represents the fraction of the energy deposited by the ion that contributes to killing in the ion-kill mode and that a fraction $(1-P)$ of the energy deposited acts in the gamma-kill mode. Thus the gamma-kill dose from a fluence $F$ of ions of stopping power $L$ is $(1-P) F L$. This dose contributes to killing of those cells in the gaps of a single particle track which survive the ion-kill mode.

Particles of high nuclear charge may generate $\delta$ rays of sufficient number and range to activate remote targets, giving their tracks the appearance of a "hairy rope" in what is called the "track-width regime." (In nuclear emulsions the track width is measured with a photodensitometer to characterize these tracks.) Here the fraction of the dose in the gamma-kill mode is small and is neglected to a first approximation. In the track-width regime, tracks in nuclear emulsions assume the appearance of a sharpened pencil as ions approach the end of their range. Here one speaks of "thindown," which arises from a kinematic limit on the energy and hence the range of $\delta$ rays, because a light particle at rest can acquire at most twice the speed of a heavy particle which collides with it, although the slowing particle generates an ever-increasing number of $\delta$ rays and an increasing stopping power. The decreased cross-sectional area of the region penetrated by $\delta$ rays leads to a limit on the interaction cross section, which cannot exceed the cross-sectional area of the region in which energy is deposited. The level of dose within this region depends on the charge and speed of the ion. These limitations are then reflected in a decreasing interaction cross section as the ion approaches the end of its range, although the cross section is different for different ions at the same speed, even in the thindown region. Thindown is observed with all detectors. It is not directly related to the size of the targets but rather to their radiosensitivity. It is not related to the Bragg peak in energy deposition. Thindown occurs only in the track-width regime. When cellular ion-kill cross sections are plotted against LET, they typically increase through (and somewhat beyond) a plateau value which approximates the cross-sectional area of the sensitive sites and then decreases abruptly, taking on the appearance of a hook, by virtue of thindown.

\section{EQUATIONS OF THE MODEL}

These considerations result in a simple set of equations which describe many radiobiological end points observed with $\gamma$ rays and with heavy-ion beams in track-segment irradiations. From a few such irradiations we extract a set of four cellular radiosensitivity parameters which then may be used to predict the response to a wide range of irradiations, including those from neutrons (of sufficiently high energy) and mixed radiation fields, provided that the particle-energy spectrum is known. Such predictions cannot be made from dose even when supported by LET. From these equations one may find the relative biological effectiveness (RBE) of an irradiation by calculating the dose of heavy ions which leads to a specific surviving fraction and the dose of $\gamma$ rays which leads to the same surviving fraction.

The radial distribution of dose represents an average over many ions of the energy deposited by $\delta$ rays in nested cylindrical shells whose axis is the ion's path. This dose falls off quadratically with radial distance to a limit determined by the maximal radial penetration of $\delta$ rays. Because of the radial dose gradient we average the dose experienced by a target at radial distances less than about three target radii. Beyond that distance it suffices to represent the average dose experienced by a target as that at the position of the center of the target (1).

If $\bar{D}$ represents the dose deposited by $\delta$ rays in a target of radius $a_{0}$ whose center is at radial distance $t$ from the ion's path, and $D_{0}$ is the dose of $\gamma$ rays at which there is an average of one hit per target, we find the ion-kill cross section by radial integration of the probability of inactivation as 


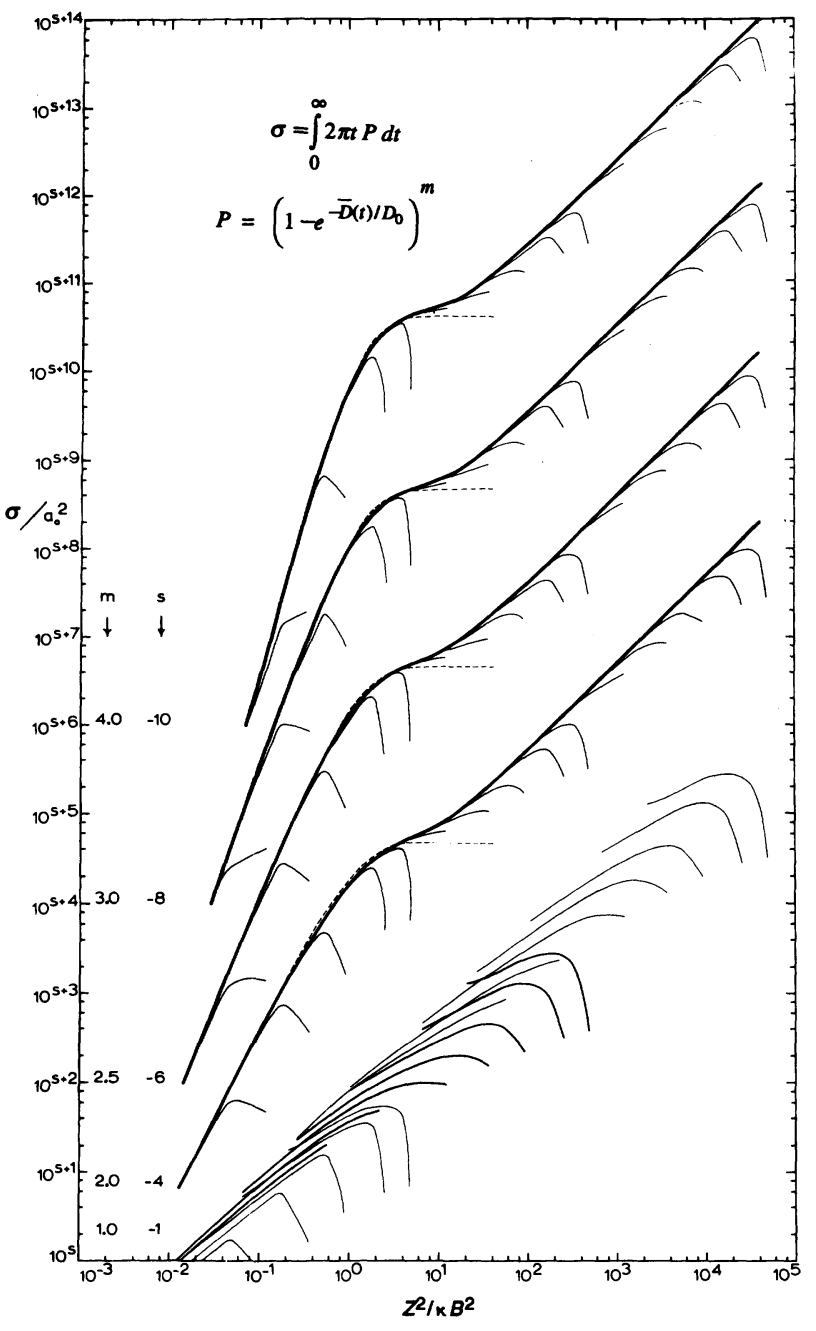

FIG. 1. $\sigma / \mathrm{a}_{0}{ }^{2}$ is plotted against $Z^{* 2} / \kappa \beta^{2}$ for constant values of $Z(1,2,5$, $10,20,50,100)$ and varying $\beta$, and $m=1,2,2.5,3$ and 4 . The family of curves plotted for $m=1$ and $D_{0}=10^{2}$ Gy and $a_{0}=10^{-4}, 10^{-5}$ and $10^{-6} \mathrm{~cm}$ are displaced both horizontally and vertically with variation in $a_{0}$, because of the plotted parameters. At higher values of $m$ there is substantial overlap of curves calculated for different values of $D_{0}$ and $a_{0}$ except for values of $\beta$ below 0.10 . The hooked-shaped branching shown in the $m$ $=1$ set of curves is repeated at higher $m$, at the same values of $Z^{* 2} / \kappa \beta^{2}$. For $m=2,2.5,3$ and 4, the curves calculated for $D_{0}=10^{6} \mathrm{~Gy}$ and $a_{0}=$ $10^{-7} \mathrm{~cm}$; for $D_{0}=10^{5} \mathrm{~Gy}$ and $a_{0}=10^{-5}, 10^{-6}$ and $10^{-7} \mathrm{~cm}$ all overlap within the confines of the heavy lines as do $D_{0}=10^{2}$ Gy and $a_{0}=10^{-4}, 10^{-5}$ and $10^{-6} \mathrm{~cm}$ where $\beta>0.10$ and for $D_{0}=10^{0}$ Gy and $a_{0}=10^{-4}$ and $10^{-5} \mathrm{~cm}$, where $\beta>0.30$. At low values of $\beta$, large values of $a_{0}$ and low values of $D_{0}$ there tends to be a hook-shaped branch due to the limit placed on the range of $\delta$ rays by kinematics. There is no plateau associated with $a_{0}$ for $m=1$. At $m=2,2.5,3$ and 4 there is increasing overlap of the different curves, with diminishing hooked branching, and a plateau of increasing length between the grain-count and track-width regimes. In the graincount regime the curves may be approximated by one of the form [1 $\left.\exp \left(-Z^{2} / \kappa \beta^{2}\right)\right]^{\mathrm{m}}$, as shown by the dashed lines which intersect the heavy curves at $Z^{* 2} / \kappa \beta^{2}=4$, and have a "plateau value" given as $\sigma \sim 1.4 \pi a_{0}^{2}$. Beyond the "plateau value" the irradiation is in the track-width regime. There is no saturation in a plot of $\sigma$ vs $Z^{* 2} / \beta^{2}$, for any value of $m$, for such curves are monotonically increasing except as determined by the maximal radial penetration of $\delta$ rays [see Fig. 2 of ref. (3)].

$$
\sigma=\int_{0}^{\infty} 2 \pi t\left(1-e^{-\bar{D} / D_{0}}\right)^{\mathrm{m}} d t
$$

For $m$ target detectors the cross section rises to a plateau value, $\sigma_{0}$, with increase in $(Z * / \beta)^{2}$ marking the end of the grain-count regime and then increases again in the trackwidth regime, falling again in the thindown region (Fig. 1). $Z^{*}$ is the effective charge of the ion at speed $\beta c$, where $c$ is the speed of light in vacuum, given by

$$
Z^{*}=Z\left(1-e^{-125 \beta / Z^{2 / 3}}\right) .
$$

The variation of cross section in the grain-count regime with charge and speed of the ion is given by

$$
\sigma=\sigma_{0}\left(1-e^{-Z^{* 2} / \kappa \beta^{2}}\right)^{\mathrm{m}},
$$

as shown in refs. (2) and (3). Then the fraction of the dose in the ion-kill mode in the grain-count regime is

$$
P=\sigma / \sigma_{0}
$$

When $P$ exceeds 0.95 we enter the track-width regime. With increasing values of $\sigma / \sigma_{0}$ the dose in the gamma-kill mode is approximated as zero. In the grain-count regime the dose in the gamma-kill mode is

$$
D_{\gamma}=(1-P) D \text {. }
$$

The probability for survival in the ion-kill mode is

$$
\Pi_{\mathrm{i}}=e^{-\sigma \mathrm{F}} .
$$

We take the survivors of the ion-kill mode to be the initial population for the gamma-kill mode. The probability for survival in the gamma-kill mode is

$$
\Pi_{\gamma}=1-\left(1-e^{-D_{\gamma} / D_{0}}\right)^{m} .
$$

This equation also describes the fraction of cells which survive an irradiation with a dose $D_{\gamma}$ of $\gamma$ rays, and therefore may be inverted to find the dose of $\gamma$ rays which leads to a specified surviving fraction, $\Pi_{\gamma}$, for the calculation of RBE.

The surviving fraction after irradiation with a beam of heavy ions is

$$
\frac{N}{N_{0}}=\Pi_{\mathrm{i}} \times \Pi_{\gamma}
$$

Thus the surviving fraction after a track-segment bombardment with a beam of ions of fluence $F$, stopping power $L$, of atomic number $Z$ moving at relative speed $\beta$ is determined 


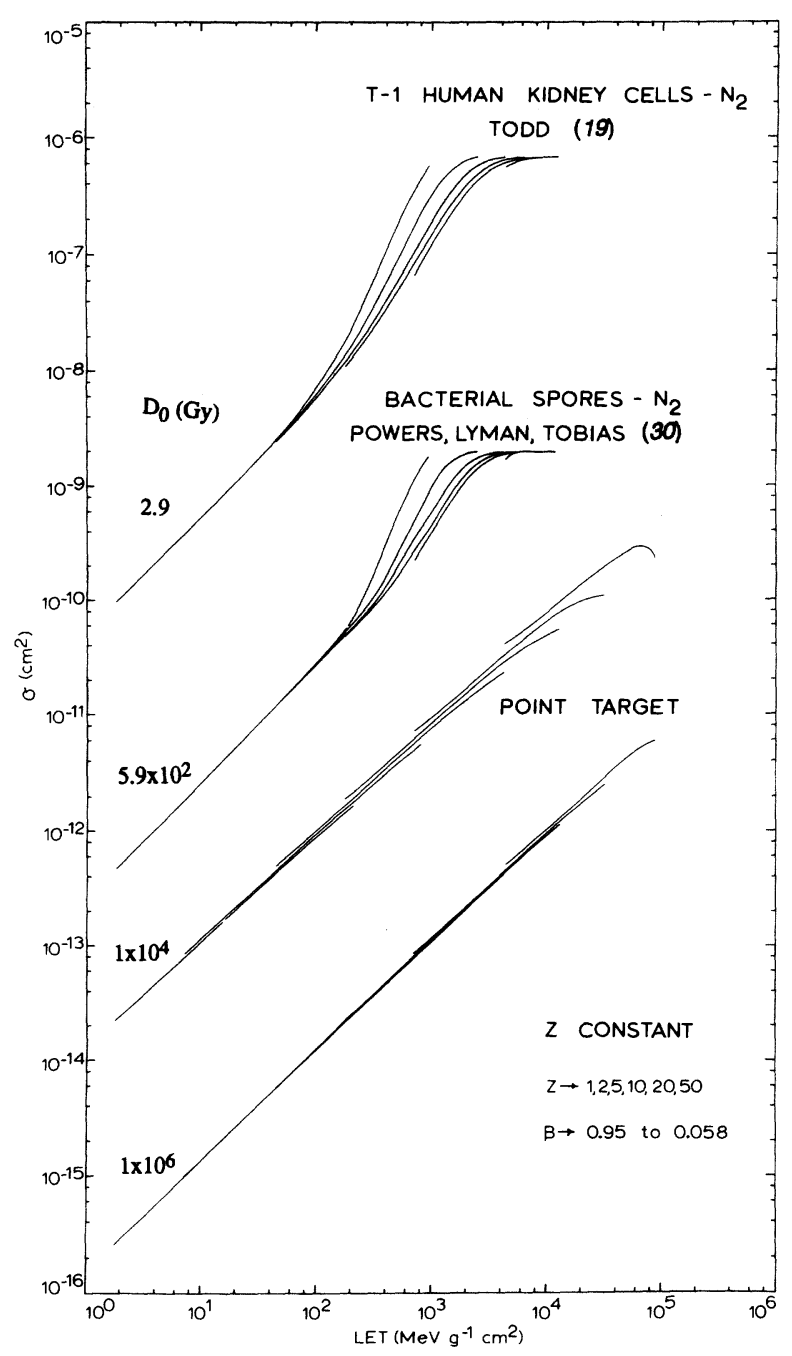

FIG. 2. Cross section vs LET for kidney cells, spores and one-hit detectors for which $D_{0}=10^{4}$ and $10^{6} \mathrm{~Gy}$, in the range of dry enzymes and viruses, plotted as continuous curves each at a single value of $Z$, with $\beta$ taking on values from 0.95 at left to 0.058 at the right-most end of each curve. The apparent saturation in cross section with increasing LET for cells is an artifact of the computation, made here only for the graincount regime. These cellular cross sections are all the extrapolated cross sections, and therefore have a contribution from the gamma-kill mode involving the interaction of secondary electrons from several ions in a beam. They cannot be analyzed by the track-segment method. At low LET, the gamma-kill mode dominates, and the curves are single-valued, with an RBE nearly equal to 1 [see Fig. 27 of ref. (32)].

from the four radiosensitivity parameters, $D_{0}, m, \kappa$ and $\sigma_{0}$, and from Eqs. (2-8).

In these calculations we have suppressed an explicit statement of the target radius, for we do not know the sizes of the biological targets. We have embedded it in a dimensionless parameter $\mathrm{k}$, whose value is determined by fitting our equations to the survival data. This parameter determines the changes in the shape of survival curves with changes in $\left(Z^{*} / \beta\right)^{2}$ of the bombarding ion. An estimate of target size is needed only when we wish to extend our calculations to the track-width regime, based on parameters determined from fits of radiobiological data to our equations in the grain-count regime. For this we make use of the equation

$$
\kappa=D_{0} a_{0}^{2} \times 10^{11} / K
$$

where $D_{0}$ is in grays, $a_{0}$ is in centimeters, and $K$ is a constant derived from the average dose deposited in an extended target by an ion passing through it. In earlier work making use of a cylindrical target whose axis is parallel to the ion's path and the radial dose model of Butts and Katz (1), the constant $K$ was set at 2 (see ref. 2). If the cylindrical target is replaced by a sphere, the constant is reduced to 0.3 . These values indicate the limits within which we are able to specify $a_{0}^{2}$ from our fitted parameters. The value of $a_{0}$ deduced from Eq. (9), the fitted value of $\kappa$ and an estimated value of $K$ (frequently taken to be 1) is used with Eq. (1) to calculate cross sections in the thindown region in the trackwidth regime.

The results of calculations of the ion-kill cross section are shown in a double logarithmic plot of cross section vs $Z^{* 2} / \kappa \beta^{2}$, in Fig. 1, for a range of values of the parameters. Typically the cross sections rise with slope $m$ to a plateau which reflects the cross-sectional area of the target, for $m>1$. The cross section then increases again until the thindown region. A detailed discussion of the parameters is given in the legend.

The final slope of a survival curve is frequently used to compute the "extrapolated cross section" from which an extrapolated RBE is calculated. These quantities are described by our equations

$$
\sigma_{(\mathrm{ext})}=\sigma_{0} P+(1-P) L / D_{0}
$$

and

$$
\operatorname{RBE}_{(\mathrm{ext})}=\left(\sigma_{0} D_{0} / L\right) P+(1-P),
$$

which are derived in ref. (2), and where "ext" refers to extrapolated values.

At high fluence as long as cell killing is in the "graincount regime," there is always a mixed contribution, principally from gamma kill at low LET, and principally from ion kill at high LET. In a double logarithmic plot of the extrapolated cross section (from the final slope of survival curves) vs LET, the slope is thus 1 at low LET and rises to $m$ at higher LET until the plateau at $\sigma_{0}$. At low LET, where gamma kill predominates, plots for different values of $Z$ overlap. At higher LET, where ion kill begins to exert its influence, these plots separate, as shown in Fig. 2, for biological cells. 
In the track-width regime there is negligible contribution from gamma kill. Our calculation of the effects then revert to Fig. 1, for the region beyond the plateau of Fig. 2. At highest LET, where thindown effects are dominant, these plots display a widely separated series of hooks with different $Z$ 's.

In Fig. 2 we plot cross sections for one-hit detectors having negligible sizes, and two different values of $D_{0}$ in the range of dry enzymes and viruses. These cross sections rise to a value determined essentially by $D_{0}$ and the maximal radial $\delta$-ray penetration. Additionally we plot calculated values of extrapolated cross sections for bacterial spores and T-1 human kidney cells (from parameters determined from fitting experimental data). As stated earlier, at lowest LET where, for cells, gamma kill predominates, the curves coalesce, while at higher LET where ion kill predominates (though still in the grain-count regime), the curves for different values of $Z$ tend to separate. This separation is sometimes not observed experimentally where only the heavier ions are used.

\section{ONE-HIT DETECTORS}

Biological one-hit detectors include dry enzymes and viruses $(1,4)$ single-strand breaks in DNA, both single- and double-strand breaks in DNA in EO buffer and three $E$. coli strains $\left(\mathrm{B}, \mathrm{B} / \mathrm{r}\right.$ and $\left.\mathrm{B}_{\mathrm{s}-1}\right)$. Additionally many physical and chemical phenomena display one-hit response, including scintillators, thermoluminescent dosimeters (TLDs), nuclear emulsions, lyoluminescence, color center formation, radical formation in alanine and the Fricke dosimeter, among others (5). The cross sections for these are also calculated from Eq. (1) with $m=1$. Cross sections increase regularly with an increase in $\left(Z^{*} / \beta\right)^{2}$ of the bombarding ion, reaching a limit determined by $D_{0}$ and the maximal radial penetration of $\delta$ rays, subsequently followed by thindown. There is no plateau cross section, and no direct relationship between target size and interaction cross section. In general we require parameters $D_{0}$ and $a_{0}$ for one-hit detectors, though for some calculations for small molecules irradiated with heavier ions the target size may be neglected entirely in a "point target" calculation. If $N$ is the number of targets per unit volume, the number of events per unit path length is $\sigma N$, and the $G$ value, the number of events per unit energy, is $\sigma N / L$.

Values of $D_{0}$ range through many orders of magnitude for different one-hit detectors. The values range from about 1-100 Gy for nuclear emulsions and from 12 to $46 \mathrm{~Gy}$ for E. coli inactivation. For single- and double-strand breaks in DNA in EO buffer we find 13 and $320 \mathrm{~Gy}$, respectively; for dry viruses inactivation values range from 4-8 kGy; for the Fricke dosimeter the value is $8 \mathrm{kGy}$; for radical formation in dry alanine we have $100 \mathrm{kGy}$; and for enzyme inactivation the values range from $40-400 \mathrm{kGy}$. To those whose interest is in mechanisms it may seem astonishing that a single model is applicable to such a range of materials and processes. The unifying theme is that a single electron transit through the target region creates changes which lead to the observed end point. This is not the case for killing or mutation or transformation or cancer induction in biological cells.

\section{C-HIT DETECTORS}

Desensitized nuclear emulsions have been shown to have a response indicative of 2-6 hits, especially when developed so as to discriminate against small latent-image sites. The production of $\mathrm{HO}_{2}$ radicals in water and of $\mathrm{H}_{2}$ in benzene display two-hit response. Supralinearity in TLDs also displays a two-hit component. For these it is assumed that the response to $\gamma$ rays follows the form of a C-or-morehit cumulative Poisson distribution rather than a multitarget model. For two-hit nuclear emulsions and two-hit chemistry $D_{0}$ lies in the neighborhood of kilograys.

\section{M-TARGET DETECTORS-BIOLOGICAL CELLS}

Radiosensitivity parameters. Our greatest interest has focused on the response of biological cells to ionizing radiations. Here it is assumed that their response to $\gamma$ rays follows the form of a multitarget model. A great variety of data obtained with $\gamma$ rays and track-segment bombardment with energetic heavy ions have been fitted to this model in which a single set of four parameters is required to fit data from all bombardments at once. Parameters fitted to data for cell killing of bacteria, bacterial spores, yeast, Chlamydomonas reinhardtii, T. confusum and artemia eggs, mammalian cells in vitro, as well as chromosome aberrations, mutation, and transformation of these cells are displayed in a numbered list in Table I. Parameter sets 1-19 are for inactivation of mammalian cells in vitro, from data obtained with track-segment heavy-ion irradiations, except for set 3 , mouse bone marrow, where data were from $\gamma$-ray and neutron bombardments and are thus defined less precisely. Data sets 21-27 are for yeast, sets 28-30 are for bacterial spores, and set 31 is for Tradescantia obtained with $\gamma$ rays and neutrons. Other sets are as labeled. Attention is called to set 34 , for an $E$. coli mutant which is not a one-hit detector, to set 37 for in vivo data for lethal mutations in C. elegans, to sets 39-41 for transformations, to set 42 for mutations in human fibroblasts, and to set 43 in which parameters are tentatively assigned to data from electron and argon-ion irradiation of rat skin, where only the shapes of the response curves rather than the number of cancers are fitted separately for the two irradiations.

Frequency distributions. To motivate mechanistic interpretations of our model, frequency distributions of parameters and of combinations of parameters are displayed in Figs. 3a-d. Intervals for these distributions are spaced 
TABLE I

A Listing of the Respective Four Parameters Required for Fitting Each Set of Experimental Data

\begin{tabular}{|c|c|c|c|c|c|c|c|c|}
\hline & Detector & Theory & Experiment & $m$ & $\kappa$ & $D_{0}(\mathrm{~Gy})$ & $\sigma_{0}\left(\mathrm{~cm}^{2}\right)$ & Comment \\
\hline 1 & $\begin{array}{l}\text { Mouse lymphoma } \\
\text { L5178Y cells, LY-S }\end{array}$ & Waligorski et al. (6) & $\begin{array}{l}\text { Jaworska et al. (7), } \\
\text { Kruszewski }{ }^{a} \text { et al. }(8-10)\end{array}$ & 2.5 & 500 & $5.00 \times 10^{-1}$ & $5.00 \times 10^{-7}$ & Aerobic survival \\
\hline 2 & $\begin{array}{l}\text { Mouse lymphoma } \\
\text { L5178Y cells, LY-R }\end{array}$ & Waligorski et al. (6) & $\begin{array}{l}\text { Jaworska et al. (7), } \\
\quad \text { Kruszewski }{ }^{a} \text { et al. }(8-10)\end{array}$ & 2.5 & 500 & $8.00 \times 10^{-1}$ & $5.00 \times 10^{-7}$ & Aerobic survival \\
\hline 3 & Mouse bone marrow & Katz and Sharma (11) & Broerse et al. (12) & 2.5 & 500 & $9.10 \times 10^{-1}$ & $4.20 \times 10^{-7}$ & Aerobic survival \\
\hline 4 & $\begin{array}{l}\text { IdU-pretreated } \\
\text { T-1 kidney cells }\end{array}$ & Roth et al. (13) & Tym and Todd (14) & 2.5 & 850 & $1.00 \times 10^{0}$ & $6.70 \times 10^{-7}$ & Aerobic survival \\
\hline 5 & Chang liver cells & Roth et al. (13) & Todd (15) & 2.5 & 900 & $1.20 \times 10^{0}$ & $4.00 \times 10^{-7}$ & Aerobic survival \\
\hline 6 & HeLa cells & Katz et al. (2) & Deering and Rice (16) & 3 & 750 & $1.40 \times 10^{0}$ & $5.60 \times 10^{-7}$ & Aerobic survival \\
\hline 7 & Leukemia cells, P388 & Katz and Sharma (3) & Berry (17) & 2.5 & 1750 & $1.55 \times 10^{0}$ & $5.80 \times 10^{-7}$ & Aerobic survival \\
\hline 8 & Human skin fibroblast & Cucinotta and $\mathrm{Katz}^{b}$ & Tsuboi et al. (18) & 3 & 680 & $1.62 \times 10^{0}$ & $8.50 \times 10^{-7}$ & Aerobic survival \\
\hline 9 & T-1 kidney cells & Katz et al. (2) & Todd (19) & 2.5 & 1000 & $1.70 \times 10^{0}$ & $6.70 \times 10^{-7}$ & Aerobic survival \\
\hline 10 & C $3 \mathrm{H} 10 \mathrm{~T} 1 / 2$ cells & Waligorski et al. (20) & Yang et al. (21) & 3 & 750 & $1.70 \times 10^{0}$ & $5.00 \times 10^{-7}$ & $\begin{array}{l}\text { Instantaneous } \\
\text { plating, aerobic } \\
\text { survival }\end{array}$ \\
\hline 11 & T-1 kidney cells & Katz et al. (2) & Barendsen et al. (22) & 2.5 & 1400 & $1.80 \times 10^{0}$ & $5.40 \times 10^{-7}$ & Aerobic survival \\
\hline 12 & $\begin{array}{l}\text { Chinese hamster cells, } \\
\text { CH2B2 }\end{array}$ & Katz and Huang (23) & Skarsgard et al. (24) & 3 & 900 & $1.82 \times 10^{0}$ & $3.00 \times 10^{-7}$ & $\begin{array}{l}\text { Abnormal } \\
\text { metaphases }\end{array}$ \\
\hline 13 & $\begin{array}{l}\text { Chinese hamster cells, } \\
\text { CH2B2 }\end{array}$ & Roth et al. (13) & Skarsgard et al. (24) & 3 & 1100 & $1.82 \times 10^{0}$ & $4.30 \times 10^{-7}$ & Aerobic survival \\
\hline 14 & $\begin{array}{l}\text { IdU-pretreated } \\
\text { T-1 kidney cells }\end{array}$ & Roth et al. (13) & Tym and Todd (14) & 2.5 & 900 & $1.90 \times 10^{0}$ & $6.70 \times 10^{-7}$ & Hypoxic survival \\
\hline 15 & $\mathrm{C} 3 \mathrm{H} 10 \mathrm{~T} 1 / 2$ cells & Waligorski et al. (20) & Yang et al. (21) & 3 & 750 & $2.80 \times 10^{0}$ & $5.00 \times 10^{-7}$ & $\begin{array}{l}\text { Delayed plating, } \\
\text { aerobic survival }\end{array}$ \\
\hline 16 & T-1 kidney cells & Katz et al. (2) & Todd (19) & 2.5 & 1300 & $2.90 \times 10^{0}$ & $6.70 \times 10^{-7}$ & Hypoxic survival \\
\hline 17 & Leukemia cells, P338 & Katz and Sharma (3) & Berry (17) & 2.5 & 2100 & $3.50 \times 10^{0}$ & $5.80 \times 10^{-7}$ & Hypoxic survival \\
\hline 18 & HeLa cells & Roth et al. (13) & Nias et al. $(25)$ & 3 & 1100 & $3.70 \times 10^{0}$ & $5.60 \times 10^{-7}$ & Hypoxic survival \\
\hline 19 & $\mathrm{~T}-1$ kidney cells & Katz et al. (2) & Barendsen et al. (22) & 2.5 & 1900 & $4.60 \times 10^{0}$ & $5.40 \times 10^{-7}$ & Hypoxic survival \\
\hline 20 & $\begin{array}{l}\text { Chinese hamster cells, } \\
\text { CH2B2 }\end{array}$ & Katz and Huang (23) & Skarsgard et al. (24) & 2 & 1400 & $2.50 \times 10^{1}$ & $6.50 \times 10^{-9}$ & $\begin{array}{l}\text { Chromatid } \\
\text { exchanges }\end{array}$ \\
\hline 21 & Nonbudding yeast & Roth et al. (13) & Raju et al. (26) & 2 & 600 & $1.00 \times 10^{1}$ & $1.00 \times 10^{-8}$ & Aerobic survival \\
\hline 22 & Haploid yeast & Katz et al. (2) & Sayeg et al. (27) & 2 & 1800 & $4.00 \times 10^{1}$ & $1.33 \times 10^{-8}$ & Aerobic survival \\
\hline 23 & Diploid yeast, BZ34 & Roth et al. (13) & Raju et al. (28) & 3 & 800 & $9.00 \times 10^{1}$ & $1.20 \times 10^{-8}$ & Aerobic survival \\
\hline 24 & Diploid yeast, BZ34 & Roth et al. (13) & Raju et al. (28) & 3 & 1200 & $1.60 \times 10^{2}$ & $1.20 \times 10^{-8}$ & Hypoxic survival \\
\hline 25 & Budding yeast & Roth et al. (13) & Raju et al. (28) & 2 & 2000 & $2.30 \times 10^{2}$ & $8.50 \times 10^{-9}$ & Aerobic survival \\
\hline 26 & Diploid yeast, $\mathrm{X} 841$ & Katz and Sharma (3) & Mortimer et al. (29) & 3 & 1100 & $2.50 \times 10^{2}$ & $3.50 \times 10^{-9}$ & Aerobic survival \\
\hline 27 & Diploid yeast, X841 & Katz and Sharma (3) & Mortimer et al. (29) & 3 & 1600 & $5.00 \times 10^{2}$ & $3.50 \times 10^{-9}$ & Hypoxic survival \\
\hline 28 & Bacterial spores & Katz et al. (2) & Powers et al. $(30)$ & 4 & 800 & $4.70 \times 10^{2}$ & $2.01 \times 10^{-9}$ & Aerobic survival \\
\hline 29 & Bacterial spores & Katz et al. (2) & Powers et al. (30) & 4 & 950 & $5.90 \times 10^{2}$ & $2.01 \times 10^{-9}$ & Hypoxic survival \\
\hline 30 & Bacterial spores & Katz et al. (2) & Powers et al. (30) & 4 & 1100 & $1.10 \times 10^{3}$ & $2.01 \times 10^{-9}$ & $\mathrm{H}_{2} \mathrm{~S}$ survival \\
\hline 31 & Tradescantia & Katz and Huang (23) & Underbrink et al. ${ }^{c}$ & 2 & 1000 & $2.10 \times 10^{0}$ & $3.50 \times 10^{-7}$ & Survival \\
\hline 32 & $T$. confusum eggs & Roth et al. (13) & Yang et al. ${ }^{d}$ & 2 & 1200 & $3.40 \times 10^{0}$ & $6.50 \times 10^{-7}$ & Aerobic survival \\
\hline 33 & Artemia eggs & Roth et al. (13) & Easter and Hutchinson (31) & 5 & 300 & $9.50 \times 10^{2}$ & $4.30 \times 10^{-10}$ & Aerobic survival \\
\hline 34 & E. coli WP2 hcr+ & Roth et al. (13) & Munson $^{e}$ & 2 & 700 & $3.80 \times 10^{1}$ & $3.80 \times 10^{-9}$ & Aerobic survival \\
\hline 35 & $\begin{array}{l}\text { Chlamydomonas rein., } \\
\text { haploid }\end{array}$ & Katz et al. (32) & Davies et al. (33) & 3 & 1400 & $1.45 \times 10^{1}$ & $5.00 \times 10^{-8}$ & Aerobic survival \\
\hline 36 & $\begin{array}{l}\text { Chlamydomonas rein., } \\
\text { diploid }\end{array}$ & Katz et al. (32) & Davies et al. (33) & 4 & 1450 & $4.20 \times 10^{1}$ & $5.40 \times 10^{-8}$ & Aerobic survival \\
\hline 37 & Caenorhabditis elegans & Cucinotta et al. (34) & Nelson et al. (35) & 2 & 750 & $6.80 \times 10^{1}$ & $2.50 \times 10^{-9}$ & Lethal mutation \\
\hline 38 & M. radiodurans & Katz et al. (32) & Dewey $(36)$ & 6 & 2650 & $1.25 \times 10^{3}$ & $5.00 \times 10^{-10}$ & Aerobic survival \\
\hline 39 & $\mathrm{C} 3 \mathrm{H} 10 \mathrm{~T} 1 / 2$ cells & Waligorski et al. (20) & Yang et al. (21) & 2 & 750 & $1.80 \times 10^{2}$ & $1.15 \times 10^{-10}$ & $\begin{array}{l}\text { Instantaneous } \\
\text { plating, } \\
\text { transformation }\end{array}$ \\
\hline 40 & $\mathrm{C} 3 \mathrm{H} 10 \mathrm{~T} 1 / 2$ cells & Waligorski et al. (20) & Yang et al. (21) & 2 & 750 & $2.60 \times 10^{2}$ & $1.15 \times 10^{-10}$ & $\begin{array}{l}\text { Delayed plating, } \\
\text { transformation }\end{array}$ \\
\hline 41 & C $3 \mathrm{H} 10 \mathrm{~T} 1 / 2$ cells & Waligorski et al. (20) & Yang et al. (21) & 3 & 475 & $5.00 \times 10^{1}$ & $7.00 \times 10^{-11}$ & $\begin{array}{l}\text { Instantaneous } \\
\text { plating, } \\
\text { transformaton } \\
\text { (alternate } \\
\text { parameters) }\end{array}$ \\
\hline 42 & Human skin fibroblast & Cucinotta and $\mathrm{Katz}^{b}$ & Tsuboi et al. (18) & 3 & 550 & $1.40 \times 10^{2}$ & $4.00 \times 10^{-11}$ & HGPRT mutation \\
\hline 43 & Cancer in rat skin & Unpublished & Burns and Albert (37) & 3 & 475 & $5.00 \times 10^{1}$ & $7.00 \times 10^{-11}$ & \\
\hline
\end{tabular}

Note. The numbers in parentheses in the columns labeled "Experiment" and "Theory" are the experimental and theoretical references.

${ }^{a}$ Unpublished results.

${ }^{b}$ Unpublished results.

${ }^{c}$ A. G. Underbrink, J. Huczkowski, B. Woch, E. Gedlek, A. Cebulska-Wasilweska, M. Litwiniszyn and E. Kasper, The Relationship of Different Somatic Mutations Induced by Neutrons and X-rays to Loss of Reproductive Integrity in Tradescantia Stamen Hairs. Report 1039/B, Institute of Nuclear Physics, Krakow, 1978.

${ }^{d}$ C. Yan, L. Craise, G. P. Welch and C. A. Tobias, Initial studies on OER, RBE and development of tribolium eggs using an oxgen beam. In Radiobiological Experiments Using Accelerated Heavy Ions at the Beavatron. LBL-2016, 1973.

${ }^{e}$ Private communication, 1973. 
a

\begin{tabular}{|l|r|r|r|r|r|r|r|r|r|r|r|r|r|l|}
\hline $6.81 \times 10^{-07}$ & 8 & & & & & & & & & & & & & \\
\hline $4.64 \times 10^{-07}$ & 1 & 2 & 4 & 6 & 7 & 9 & 10 & 11 & 14 & 15 & 16 & 17 & 18 & 19 \\
\hline $3.16 \times 10^{-07}$ & 3 & 5 & 13 & 31 & & & & & & & & & & \\
\hline $2.15 \times 10^{-07}$ & 12 & & & & & & & & & & & & & \\
\hline $1.47 \times 10^{-07}$ & & & & & & & & & & & & & & \\
\hline $1.00 \times 10^{-07}$ & & & & & & & & & & & & & & \\
\hline $6.81 \times 10^{-08}$ & & & & & & & & & & & & & & \\
\hline $4.64 \times 10^{-08}$ & 35 & 36 & & & & & & & & & & & & \\
\hline $3.16 \times 10^{-08}$ & & & & & & & & & & & & & & \\
\hline $2.15 \times 10^{-08}$ & & & & & & & & & & & & & & \\
\hline $1.47 \times 10^{-08}$ & & & & & & & & & & & & & & \\
\hline $1.00 \times 10^{-08}$ & 21 & 22 & 23 & 24 & & & & & & & & & & \\
\hline $6.81 \times 10^{-09}$ & 25 & & & & & & & & & & & & & \\
\hline $4.64 \times 10^{-09}$ & 20 & & & & & & & & & & & & & \\
\hline $3.16 \times 10^{-09}$ & 26 & 27 & 34 & & & & & & & & & & & \\
\hline $2.15 \times 10^{-09}$ & 37 & & & & & & & & & & & & & \\
\hline $1.47 \times 10^{-09}$ & 28 & 29 & 30 & & & & & & & & & & & \\
\hline $1.00 \times 10^{-09}$ & & & & & & & & & & & & & & \\
\hline $6.81 \times 10^{-10}$ & & & & & & & & & & & & & & \\
\hline $4.64 \times 10^{-10}$ & 38 & & & & & & & & & & & & & \\
\hline $3.16 \times 10^{-10}$ & 33 & & & & & & & & & & & & & \\
\hline $2.15 \times 10^{-10}$ & & & & & & & & & & & & & & \\
\hline $1.47 \times 10^{-10}$ & & & & & & & & & & & & & & \\
\hline $1.00 \times 10^{-10}$ & 39 & 40 & & & & & & & & & & & & \\
\hline $6.81 \times 10^{-11}$ & 41 & 43 & & & & & & & & & & & & \\
\hline $4.64 \times 10^{-11}$ & & & & & & & & & & & & & & \\
\hline $3.16 \times 10^{-11}$ & 42 & & & & & & & & & & & & & \\
\hline $2.15 \times 10^{-11}$ & & & & & & & & & & & & & & \\
\hline $1.47 \times 10^{-11}$ & & & & & & & & & & & & & & \\
\hline $1.00 \times 10^{-11}$ & & & & & & & & & & & & & & \\
\hline
\end{tabular}

\section{C}

\begin{tabular}{|l|r|r|r|r|r|r|r|r|l|}
\hline $6.81 \times 10^{03}$ & & & & & & & & & \\
\hline $4.64 \times 10^{03}$ & & & & & & & & & \\
\hline $3.16 \times 10^{03}$ & & & & & & & & & \\
\hline $2.15 \times 10^{03}$ & & & & & & & & & \\
\hline $1.47 \times 10^{03}$ & & & & & & & & & \\
\hline $1.00 \times 10^{03}$ & 1 & 7 & & & & & & & \\
\hline $6.81 \times 10^{02}$ & 4 & 5 & 11 & & & & & & \\
\hline $4.64 \times 10^{02}$ & 2 & 3 & 6 & 9 & 12 & 13 & 14 & 17 & 31 \\
\hline $3.16 \times 10^{02}$ & 8 & 10 & 16 & 19 & 32 & & & & \\
\hline $2.15 \times 10^{02}$ & 15 & 18 & & & & & & & \\
\hline $1.47 \times 10^{02}$ & & & & & & & & & \\
\hline $1.00 \times 10^{02}$ & & & & & & & & & \\
\hline $6.81 \times 10^{01}$ & 35 & & & & & & & & \\
\hline $4.64 \times 10^{01}$ & 20 & 21 & & & & & & & \\
\hline $3.16 \times 10^{01}$ & 22 & 36 & & & & & & & \\
\hline $2.15 \times 10^{01}$ & & & & & & & & & \\
\hline $1.47 \times 10^{01}$ & 34 & & & & & & & & \\
\hline $1.00 \times 10^{01}$ & 37 & & & & & & & & \\
\hline $6.81 \times 10^{00}$ & 23 & 24 & 25 & 41 & 43 & & & & \\
\hline $4.64 \times 10^{00}$ & & & & & & & & & \\
\hline $3.16 \times 10^{00}$ & 26 & 27 & 39 & 42 & & & & & \\
\hline $2.15 \times 10^{00}$ & 40 & & & & & & & & \\
\hline $1.47 \times 10^{00}$ & 28 & 29 & 38 & & & & & & \\
\hline $1.00 \times 10^{00}$ & 30 & & & & & & & & \\
\hline $6.81 \times 10^{-01}$ & & & & & & & & & \\
\hline $4.64 \times 10^{-01}$ & & & & & & & & & \\
\hline $3.16 \times 10^{-01}$ & 33 & & & & & & & & \\
\hline $2.15 \times 10^{-01}$ & & & & & & & & & \\
\hline $1.47 \times 10^{-01}$ & & & & & & & & & \\
\hline $1.00 \times 10^{-01}$ & & & & & & & & & \\
\hline
\end{tabular}

b

\begin{tabular}{|l|l|l|l|l|l|l|l|l|l|}
\hline $6.81 \times 10^{03}$ & & & & & & & & & \\
\hline $4.64 \times 10^{03}$ & & & & & & & & & \\
\hline $3.16 \times 10^{03}$ & & & & & & & & & \\
\hline $2.15 \times 10^{03}$ & & & & & & & & & \\
\hline $1.47 \times 10^{03}$ & & & & & & & & & \\
\hline $1.00 \times 10^{03}$ & 30 & 38 & & & & & & & \\
\hline $6.81 \times 10^{02}$ & 33 & & & & & & & & \\
\hline $4.64 \times 10^{02}$ & 27 & 28 & 29 & & & & & & \\
\hline $3.16 \times 10^{02}$ & & & & & & & & & \\
\hline $2.15 \times 10^{02}$ & 25 & 26 & 40 & & & & & & \\
\hline $1.47 \times 10^{02}$ & 24 & 39 & & & & & & & \\
\hline $1.00 \times 10^{02}$ & 42 & & & & & & & & \\
\hline $6.81 \times 10^{01}$ & 23 & & & & & & & & \\
\hline $4.64 \times 10^{01}$ & 37 & 41 & 43 & & & & & & \\
\hline $3.16 \times 10^{01}$ & 22 & 34 & 36 & & & & & & \\
\hline $2.15 \times 10^{01}$ & 20 & & & & & & & & \\
\hline $1.47 \times 10^{01}$ & & & & & & & & & \\
\hline $1.00 \times 10^{01}$ & 21 & 35 & & & & & & & \\
\hline $6.81 \times 10^{00}$ & & & & & & & & & \\
\hline $4.64 \times 10^{00}$ & & & & & & & & & \\
\hline $3.16 \times 10^{00}$ & 17 & 18 & 19 & 32 & & & & & \\
\hline $2.15 \times 10^{00}$ & 15 & 16 & & & & & & & \\
\hline $1.47 \times 10^{00}$ & 7 & 8 & 9 & 10 & 11 & 12 & 13 & 14 & 31 \\
\hline $1.00 \times 10^{00}$ & 4 & 5 & 6 & & & & & & \\
\hline $6.81 \times 10^{-01}$ & 2 & 3 & & & & & & & \\
\hline $4.64 \times 10^{-01}$ & 1 & & & & & & & & \\
\hline $3.16 \times 10^{-01}$ & & & & & & & & & \\
\hline $2.15 \times 10^{-01}$ & & & & & & & & & \\
\hline $1.47 \times 10^{-01}$ & & & & & & & & & \\
\hline $1.00 \times 10^{-01}$ & & & & & & & & & \\
\hline
\end{tabular}

\section{d}

\begin{tabular}{|l|r|r|r|r|r|r|r|r|l|}
\hline $6.81 \times 10^{-09}$ & & & & & & & & & \\
\hline $4.64 \times 10^{-09}$ & & & & & & & & & \\
\hline $3.16 \times 10^{-09}$ & & & & & & & & & \\
\hline $2.15 \times 10^{-09}$ & & & & & & & & & \\
\hline $1.47 \times 10^{-09}$ & 8 & 15 & 16 & 18 & 24 & 30 & 32 & 36 & \\
\hline $1.00 \times 10^{-09}$ & 6 & 9 & 10 & 14 & 19 & 23 & 27 & 28 & 29 \\
\hline $6.81 \times 10^{-10}$ & 2 & 3 & 4 & 11 & 13 & 17 & 25 & 26 & 31 \\
\hline $4.64 \times 10^{-10}$ & 1 & 5 & 7 & 12 & 35 & & & & \\
\hline $3.16 \times 10^{-10}$ & & & & & & & & & \\
\hline $2.15 \times 10^{-10}$ & 22 & 37 & 38 & & & & & & \\
\hline $1.47 \times 10^{-10}$ & 21 & 34 & & & & & & & \\
\hline $1.00 \times 10^{-10}$ & 20 & & & & & & & & \\
\hline $6.81 \times 10^{-11}$ & & & & & & & & & \\
\hline $4.64 \times 10^{-11}$ & & & & & & & & & \\
\hline $3.16 \times 10^{-11}$ & 40 & & & & & & & & \\
\hline $2.15 \times 10^{-11}$ & 39 & & & & & & & & \\
\hline $1.47 \times 10^{-11}$ & & & & & & & & & \\
\hline $1.00 \times 10^{-11}$ & 42 & & & & & & & & \\
\hline $6.81 \times 10^{-12}$ & 41 & 43 & & & & & & & \\
\hline $4.64 \times 10^{-12}$ & & & & & & & & & \\
\hline $3.16 \times 10^{-12}$ & & & & & & & & & \\
\hline $2.15 \times 10^{-12}$ & & & & & & & & & \\
\hline $1.47 \times 10^{-12}$ & & & & & & & & & \\
\hline $1.00 \times 10^{-12}$ & & & & & & & & & \\
\hline
\end{tabular}

FIG. 3. Panel a: Frequency distribution of the parameter $\sigma_{0}$, in $\mathrm{cm}^{2}$. Panel b: Frequency distribution of the parameter $D_{0}$, in gray. Panel c: Frequency distribution of the parameter combination $\kappa / D_{0}$, proportional to $a_{0}{ }^{2} \cdot \kappa$ is dimensionless and $D_{0}$ is in gray. Panel d: Frequency distribution of the parameter combination $D_{0} \sigma_{0} / \kappa$, proportional to $\sigma_{0} / a_{0}^{2} . D_{0}$ is in gray, $\sigma_{0}$ is in $\mathrm{cm}^{2}$, and $\kappa$ is dimensionless. Intervals are spaced according to the sixth root of 10 . The number in each box refers to the data set numbers in Table $I$. 

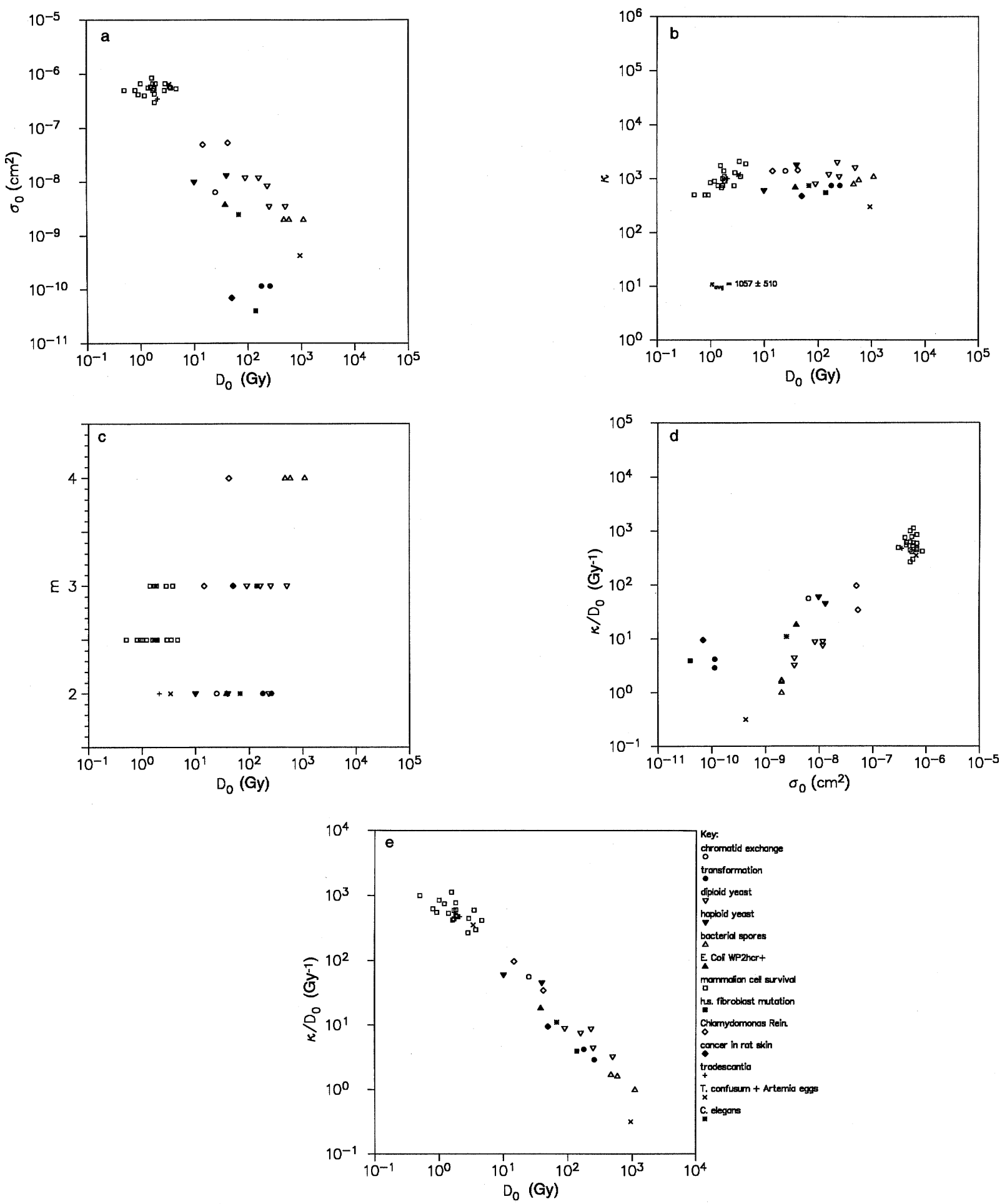

FIG. 4. Panel a: A display of the correlation between the parameters $D_{0}$ and $\sigma_{0}$, where $D_{0}$ is in gray and $\sigma_{0}$ is in $\mathrm{cm}^{2}$. Panel b: A display of the correlation between the parameters $D_{0}$ and $\kappa$, where $D_{0}$ is in gray and $\kappa$ is dimensionless. Panel c: A display of the correlation between the parameters $D_{0}$ and $m$, where $D_{0}$ is in gray and $m$ is dimensionless. Panel d: A display of the correlation between the parameter $\sigma_{0}$ and the combination of parameters $\kappa / D_{0}$, proportional to $a_{0}^{2} . D_{0}$ is in gray and $\kappa$ is dimensionless. Panel e: A display of the correlation between the parameters $D_{0}$ and the combination of parameters $\kappa / D_{0}$, proportional to $a_{0}^{2} . D_{0}$ is in gray and $\kappa$ is dimensionless. 
according to the sixth root of 10 , as a compromise between resolution and smoothness for data spread over several decades. For identification the number in each box refers to the data sets of Table I. The parameter $\sigma_{0}$ is displayed in Fig. 3a. The data for mammalian cell killing are contained in a narrow peak. Data for other end points are grouped similarly. The distribution of $D_{0}$ is displayed in Fig. 3b. Groups of parameters are displayed in Figs. $3 \mathrm{c}$ and $3 \mathrm{~d}$, as $\kappa / D_{0}$, proportional to the square of the radius of the presumed internal target, and as $D_{0} \sigma_{0} / \kappa$, proportional to the quotient of the plateau value of the cross section and the square of the radius of the internal target, respectively. Our calculations presently give only a range of values for the proportionality constant depending on whether the target is presumed to be a cylinder whose axis is parallel to the ion's path or a sphere, and on the details of the calculation of the radial dose distribution. The frequency distribution of parameters may also be useful if one wishes to select a typical set of cellular parameters for purposes of radiation protection or health physics, in the event that calculations from this model are seen to supplement or replace "quality factors."

Plots of relationships among parameters. Some of our parameters are closely correlated. To display these relationships we plot Figs. 4a-e. We plot $D_{0}$ vs $\sigma_{0}$ in Fig. 4a. Here the coefficient of correlation taking all points into account is -0.85 . In Fig. $4 \mathrm{~b}$ we plot $D_{0}$ vs $\kappa$. In Fig. $4 \mathrm{c}$ we plot $D_{0}$ vs $m$. In Fig. $4 \mathrm{~d}$ we plot $\sigma_{0}$ vs $\kappa / D_{0}$. Here the coefficient of correlation is 0.85 , taking all points into account. Since $\kappa / D_{0}$ is proportional to $a_{0}^{2}$, this plot relates the square of the radius of the internal target to the plateau value of the activation cross section. In the initial construction of this model the action cross section $\sigma$ was taken to be proportional to the model cross section calculated for the internal target, but no quantitative relationship was assumed (see ref. 2). Finally, in Fig. $4 \mathrm{e}$ we plot $D_{0}$ vs $\kappa / D_{0}$, relating the dose at which there is one hit per target to the square of the radius of the internal targets. Here the coefficient of correlation is -0.98 .

\section{CONCLUSIONS}

Results presented here summarize some 25 years of study of radiobiological data. A single four-parameter model is used to fit data for a wide range of biological test objects and end points, as reported by many investigators using different facilities.

Radiosensitivity parameters $D_{0}$ and $\sigma_{0}$, determined principally by $\gamma$ irradiation and heavy-ion irradiation, respectively, spread over 4 orders of magnitude as shown in Figs. $3 a$ and $3 b$. The parameter $\kappa$, which essentially determines the location in LET of the peak of a plot of RBE vs LET, barely spreads over a single order of magnitude. There are distinct correlations between $D_{0}$ and $\sigma_{0}$, and among $\sigma_{0}, D_{0}$ and $\kappa / D_{0}$. It is remarkable that these correla- tions hold for the great variety of species and end points described in these data.

Radiobiologists are concerned with mechanisms. Indeed, this has been much of the motivation for these extensive studies of the response to high-LET radiation. Here we show that the variation of response with LET is primarily physical in origin. The present model is mechanistic only in its description of track structure and in its choice of parameters. Hopefully, those studying biological mechanisms will see this work as simplifying their task, possibly now redirected toward explaining the biological basis for this choice of parameters, and the numerical values they display for different species and end points.

\section{ACKNOWLEDGMENT}

This work is supported by the U.S. Department of Energy, Grant No. DE-FG02-88ER60634.

Received: July 13, 1993; accepted: June 30, 1994

\section{REFERENCES}

1. J. J. Butts and R. Katz, Theory of RBE for heavy ion bombardment of dry enzymes and viruses. Radiat. Res. 30, 855-871 (1967).

2. R. Katz, B. Ackerson, M. Homayoonfar and S. C. Sharma, Inactivation of cells by heavy ion bombardment. Radiat. Res. 47, 402-405 (1971).

3. R. Katz and S. C. Sharma, Response of cells to fast neutrons, stopped pions and heavy ion beams. Nucl. Instrum. Methods 110, 92-116 (1973).

4. M. P. R. Waligorski, R. N. Hamm and R. Katz, Inactivation of dry enzymes and viruses by energetic heavy ions. Radiat. Phys. Chem. 30, 201-208 (1987).

5. R. Katz, Track structure theory in radiobiology and in radiation detection. Nucl. Track Detect. 2, 1-28 (1978).

6. M. P. R. Waligorski, M. Kruszewski, E. A. Krasavin, J. V. Ovodkov and O. Rosiek, Track structure analysis of survival of two mouse lymphoma L5178Y cell strains of different radiation sensitivity. Radiat. Prot. Dosim. 52, 207-210 (1994).

7. A. Jaworska, J. V. Ovodkov, V. M. Nazarov, O. Rosiek, Z. Sawicki and $\mathrm{M}$. Zielczynski, The influence of radiation quality on survival of two mouse lymphoma L5178Y cell strains of different radiation sensitivity. Nukleonika 33, 247-255 (1988).

8. M. Kruszewski, J. V. Ovodkov, V. N. Gerasimenko, A. L. Karpovski, $\mathrm{O}$. Rosiek and Z. Sawicki, The influence of radiation quality on survival of two mouse lymphoma L5178Y cell strains of different radiation sensitivity. 2. Effects of $9 \mathrm{GeV}$ protons. Nukleonika 33, 339-343 (1988).

9. M. Kruszewski, J. V. Ovodkov, E. A. Krasavin, P. N. Lobachevsky, O. Rosiek, Z. Sawicki and A. P. Tcherevatenko, The influence of radiation quality on survival of two mouse lymphoma L5178Y cell strains of different radiation sensitivity. 4. Effects of $2.5 \mathrm{MeV}$ helium ions. Nukleonika 34, 109-114 (1989).

10. M. Kruszewski, J. V. Ovodkov, E. A. Krasavin, P. N. Lobachevsky, O. Rosiek, Z. Sawicki and A. P. Tcherevatenko, The influence of radiation quality on survival of two mouse lymphoma L5178Y cell strains of different radiation sensitivity. 5. Effects of $1.6 \mathrm{MeV}$ deuterons. Nukleonika 34, 315-321 (1989).

11. R. Katz and S. C. Sharma, RBE-dose relations for neutrons and pions. Phys. Med. Biol. 20, 410-419 (1975). 
12. J. J. Broerse, A. C. Engels, P. LeLieveld, L. M. van Putten, W. Duncan, D. Greene, J. B. Massey, C. W. Gilbert, J. H. Hendry and A. Howard, The survival of colony-forming units in mouse bonemarrow after in vivo irradiation with D-T neutrons, X- and gammaradiation. Int. J. Radiat. Biol. 19, 101-110 (1971).

13. R. A. Roth, S. C. Sharma and R. Katz, Systematic evaluation of cellular radiosensitivity parameters. Phys. Med. Biol. 21, 491-503 (1976).

14. R. Tym and P. W. Todd, The sensitization by iododeoxyuridine of cultured human cells to the lethal effect of X-rays and heavy ions. Int. J. Radiat. Biol. 8, 589-604 (1964).

15. P. W. Todd, Heavy ion irradiation of human and Chinese hamster cells in vitro. Radiat. Res. 61, 288-297 (1975).

16. R. A. Deering and R. Rice, Jr., Heavy ion irradiation of HeLa cells Radiat. Res. 17, 774-786 (1962).

17. R. J. Berry, Survival of murine leukemia cells in vivo after irradiation in vitro under aerobic and hypoxic conditions with monoenergetic accelerated charged particles. Radiat. Res. 44, 237-247 (1970).

18. K. Tsuboi, T. C. Yang and D. J. Chen, Cytotoxic and mutagenic effects of high-LET charged iron particles on human skin fibroblasts. Radiat. Res. 129, 171-176 (1992).

19. P. Todd, Heavy-ion inactivation of cultured human cells. Radiat. Res. Suppl. 7, 196-207 (1967)

20. M. P. R. Waligorski, G. L. Sinclair and R. Katz, Radiosensitivity parameters for neoplastic transformation in C3H10T1/2 cells. Radiat. Res. 111, 424-437 (1987).

21. T. C. Yang, L. M. Craise and C. A. Tobias, Neoplastic cell transformation by heavy charged particles. Radiat. Res. 104, S177-S187 (1985).

22. G. W. Barendsen, C. J. Koot, G. R. van Kersen, D. K. Bewley, S. B. Field and C. J. Parnell, The effect of oxygen on impairment of the proliferative capacity of human cells in culture by ionizing radiation of different LET. Int. J. Radiat. Biol. 10, 317-327 (1966).

23. R. Katz and G. Huang, Radiosensitivity parameters for cell survival in Tradescantia and for chromosome aberrations in Chinese hamster cells. Radiat. Prot. Dosim. 31, 261-263 (1990).

24. L. D. Skarsgard, B. A. Kihlman, I. Parker, C. M. Pujara and S. Richardson, Survival, chromosome abnormalities, and recovery in heavy-ion and X-irradiated mammalian cells. Radiat. Res. Suppl. 7, 208-221 (1967).

25. A. H. W. Nias, D. Greene, M. Fox and R. L. Thomas, Effect of $14 \mathrm{MeV}$ monoenergetic neutrons on $\mathrm{HeLa}$ and $\mathrm{p} 388 \mathrm{~F}$ cells in vitro. Int. J. Radiat. Biol. 13, 449-456 (1967).
26. M. R. Raju, M. Gnanapurani, B. Stackler, U. Madhvanath, J. Howard, J. T. Lyman, T. R. Manney and C. A. Tobias, Influence of linear energy transfer on the radioresistance of budding haploid yeast cells. Radiat. Res. 51, 310-317 (1972).

27. J. A. Sayeg, A. C. Birge, C. A. Beam and C. A. Tobias, The effects of accelerated carbon nuclei and other radiations on the survival of haploid yeast. II. Biological experiments. Radiat. Res. 10, 449-461 (1959).

28. M. R. Raju, M. Gnanapurani, B. Stackler, B. T. Martins, U. Madhvanath, J. Howard, J. T. Lyman and R. K. Mortimer, Induction of heteroallelic reversions and lethality in Saccharomyces cerevisiae exposed to radiations of various LET $\left({ }^{60} \mathrm{Co} \gamma\right.$ rays, heavy ions and $\pi^{-}$mesons) in air and nitrogen atmospheres. Radiat. Res. 47, 635-643 (1971).

29. R. Mortimer, T. Brustad and D. V. Cormack, Influence of linear energy transfer and oxygen tension on the effectiveness of ionizing radiations for induction of mutations and lethality in Saccharomyces cerevisiae. Radiat. Res. 26, 465-482 (1965).

30. E. L. Powers, J. T. Lyman and C. A. Tobias, Some effects of accelerated charged particles on bacterial spores. Int. J. Radiat. Biol. 14, 313-330 (1968).

31. S. S. Easter, Jr. and F. Hutchinson, Effects of radiations of different LETs on artemia eggs. Radiat. Res. 15, 333-340 (1961).

32. R. Katz, S. C. Sharma and M. Homayoonfar, The structure of particle tracks. In Topics in Radiation Dosimetry, Suppl. 1 (F. H. Attix, Ed.), pp. 317-383. Academic Press, New York, 1972.

33. D. R. Davies, P. D. Holt and D. G. Papworth, The survival curves of haploid and diploid Chlamydomonas reinhardtii exposed to radiations of different LET. Int. J. Radiat. Biol. 15, 75-87 (1969).

34. F. A. Cucinotta, J. W. Wilson and R. Katz, Radiosensitivity parameters for mutagenesis of Caenorhabditis elegans. Radiat. Prot. Dosim. 52, 25-27 (1994).

35. G. A. Nelson, W. W. Schubert, T. M. Marshall, E. R. Benton and E. V. Benton, Radiation effects in Caenorhabditis elegans, mutagenesis by high and low LET ionizing radiation. Mutat. Res. 212, 181-192 (1989).

36. D. L. Dewey, The survival of Micrococcus radiodurans irradiated at high LET and the effect of acridine orange. Int. J. Radiat. Biol. 16, 583-592 (1969).

37. F. J. Burns and R. E. Albert, Radiation carcinogenesis in rat skin. In Radiation Carcinogenesis (A. C. Upton, R. E. Albert, F. J. Burns and R. E. Shore, Eds.), pp. 191-214. Elsevier, New York, 1986. 